\title{
Versuche über Eiweißresorption.
}

Von

Otto Cohnheim (Heidelberg).

(Aus dem physiologisch-chemischen Laboratorium der zoologischen Station in Neapel.)

(Der Redaktion zugegangen am 15. März 1909.)

Die Frage, in welcher Form die Eiweißkörper der Nahrung, nachdem die Verdauungssekrete auf sie eingewirkt haben, die Darmwand durchsetzen und ins Blut eintreten, ist heute noch nicht entschieden. Eine sichere Antwort werden wir auch erst erhalten, wenn wir die Stoffe jenseits der Darmwand auffinden, und das ist beim Säugetier bekanntlich bisher niemals gelungen, hauptsächlich, weil bei dem raschen Wechsel des Blutes die gleichzeitig vorhandenen Mengen zu gering waren, um chemisch untersucht zu werden. ${ }^{1}$ ) Es lag nun nahe, dadurch weiterzukommen, daß man den isolierten, aus dem Körper entfernten Darm resorbieren ließ. An Säugetieren bin ich nicht zum Ziel gekommen, dagegen habe ich vor einer Reihe von Jahren den aus dem Körper herausgenommenen Darm von Weichtieren, Cephalopoden, in das verdünnte Blut der Tiere gelegt, mit Pepton gefüllt und eine reichliche Resorption, $d . h$. einen Übergang von stickstoffhaltigen Verbindungen in das außen befindliche Blut beobachtet $^{2}$ ) Soweit feststellbar, war der Stickstoff in Form der Aminosäuren vorhanden, wie sie bei jeder Eiweißspaltung entstehen. Jedenfalls konnte keine Synthese von Eiweiß, kein Übergang von Pepton und auch keine über die Aminosäuren herausgehende Umwandlung des Eiweißes beobachtet werden.

1) Die Literatur über die Frage habe ich zusammengesteilt und kritisch besprochen in meinen Vorlesungen über die Physiologie der Verdauung und Ernährung, S. $226 \mathrm{ff}$., Berlin 1908.

2) O. Cohnheim, Diese Zeitschrift, Bd. XXXV, S. 396 (1902).

Hoppe-Seyler's Zeitschrift f. physiol. Chemie. LIX. 
Wie weit dieser Befund auf die Wirbeltiere übertragen werden durfte, war aber fraglich, und so habe ich die Versuche an den Därmen von kaltblütigen Wirbeltieren, Fischen, wiederholt. Bei den Kaltblütern war zu erwarten, daß das isolierte Organ lange genug überlebend bleiben würde, und die Fische luden besonders zu derartigen Untersuchungen ein, weil bei ihnen der Darm im Vergleich zur Masse des Körpers auffallend klein ist, also vermutlich in der Zeiteinheit sehr intensiv arbeiten muß.

Die Versuche sind im April und Mai 1908 in der physiologischen Abteilung der zoologischen Station zu Neapel angestellt worden. Ich erlaube mir auch, an dieser Stelle dem Leiter der chemischen Abteilung, Herrn Dr. Henze, für seine Hilfsbereitschaft, und Herrn Cav. Dr. Lo Bianco für die reichliche Versorgung mit dem Material meinen verbindlichsten Dank auszusprechen.

Nachdem ich eine Reihe anderer Fischarten probiert hatte, erwies sich am geeignetsten der zur Familie der Labriden gehörige Crenilabrus pavo, an dem ich die große Mehrzahl der Versuche gemacht habe; neben ihm habe ich einige Male den zur selben Familie gehörigen und ganz gleich gebauten Labrus festivus benutzt. Crenilabrus pavo ${ }^{1}$ ) hat keinen Magen, die Speiseröhre geht direkt in den Dünndarm über, in dessen Anfangsteil die Ausführungsgänge der Leber und des in der ganzen Bauchhöhle verteilt liegenden Pankreas münden. Infolge des Fehlens des Magens ist der Dünndarm weiter und geräumiger als bei den anderen Fischen, die ja meist einen sehr großen Magen und einen kleinen Darm haben. Immerhin macht der leere Darm nur 1,5\% des Körpergewichts aus. Der Enddarm setzt sich scharf von dem übrigen Darm ab. Die Därme frisch gefangener Crenilabrus waren stets vollgestopft mit kleinen Muscheln und zwar einer einzigen Art, Mytilus minimus (nach Angabe von Dr. Lo Bianco). Während des Aufenthaltes im Aquarium wurden die Därme allmählich leer. Das Blutserum der Knochenfische ist nach den Bestimmungen

1) Literatur: J. F. Meckel, System der vergl. Anatomie, Bd. IV, S. 251 (1829). - E. Laguesse, Revue biologique du Nord de la France, Bd. VII, S. 343 (1895). 
von Dekhuyzen ${ }^{1}$ ) und von Bottazzi ${ }^{2}$ ) etwas konzentrierter als das der Säugetiere. Nach einigem Probieren fand ich, daß der Darm sehr gut in einer Lösung lebendig blieb, welche die Salze im Verhältnis der üblichen Ringerschen Lösung, aber die für $1000 \mathrm{ccm}$ berechnete Salzmenge in $800 \mathrm{ccm}$ Wasser enthielt. Das sind also im Liter 0,375 $\mathrm{g} \mathrm{NaHCO}_{3}, 0,3 \mathrm{~g} \mathrm{CaCl}_{2}$, $0,525 \mathrm{~g} \mathrm{KCl}$ und $11,25 \mathrm{~g} \mathrm{NaCl}$.

(Nachträglich teilt mir Herr Dr. Henze mit, ich hätte vermutlich bei einem Teil der Versuche statt $\mathrm{KCl} \mathrm{KClO}_{3}$ in Händen gehabt. Bei den ersten Versuchen ist das sicher nicht der Fall gewesen, und ich habe mich auch noch ausdrücklich davon überzeugt, daß $\mathrm{KClO}_{3}$ selbst in fünffach größerer Konzentration die beschriebenen Veränderungen nicht hervorruft.)

Die Versuche stellte ich folgendermaßen an: Ich tötete die Fische, indem ich hinter den Kiemen einging und das Herz durchschnitt. Das ausfließende Blut fing ich in der Ringerschen Lösung auf, defibrinierte durch Schlagen und filtrierte durch Gaze. In diese bluthaltige Lösung kam der Darm, der rasch aus dem Tiere entfernt, mit der zu resorbierenden Lösung gefüllt und an beiden Enden zugebunden wurde. Ich nahm den Darm von der Stelle unterhalb der Gallengangmündung bis zum Anfang des Enddarms. Durch die Lösung wurde während der Versuche Sauerstoff geleitet. Die Versuche dauerten 6-9Stunden. Der mit Muscheln gefüllte Darm der frisch gefangenen Fische zeigte nie eine Spur von Bewegung. Der mit Flüssigkeit gefüllte bewegte sich nach Art der Pendelbewegungen des Katzendarms, wenn auch langsamer, und ließ auch bisweilen Einschnürungen sehen, die über den Darm hinliefen. Ich überzeugte mich zunächst, daß der Darm überhaupt resorbierte, indem ich ihn mit einer sehr dünnen Lösung von Jodnatrium füllte. Nach einer Stunde war in der Außenflüssigkeit Jod nachzuweisen. Es verschwand aber nie ganz aus dem Darminhalt, wie ich das bei Oktopoden beobachtet habe. $\mathrm{Zu}$ den Resorptionsversuchen benutzte ich eine Peptonlösung, die durch weitgehende Pepsinverdauung von Milcheiweiß (Plasmon) gewonnen war,

1) M. C. Dekhuyzen, Arch. néerland. des Sciences exactes nat., 1905.

2) Fil. Bottazzi, Arch. di Fisiologia, Bd. III (1906). 
und die eine starke Biuretreaktion und die übrigen Eiweißreaktionen gab. Wenn das Pepton mit Baryumcarbonat destilliert wurde, änderte sich der Titer einer vorgelegten Schwefelsäure höchstens spurweise. Einige Male habe ich auch die mit Muscheln gefüllten Därme ohne weitere Füllung resorbieren lassen.

Am Ende des Versuches wurde die Außenflüssigkeit durch Kochen mit einigen Tropfen Essigsäure enteiweißt. Im Filtrat fand sich, ganz wie bei den Oktopoden, reichlich nach Kjeldahl bestimmbarer Stickstoff, aber keine Biuretreaktion, oder eine ganz schwache, die in keiner Weise der Stickstoffmenge entsprach. Zeigte sich überhaupt Biuretreaktion, so waren auch nicht mehr alle Därme beweglich. Am stärksten war die Biuretreaktion in einem nicht weiter untersuchten Falle, in dem der Versuch 12 Stunden gedauert hatte. Im Innern der Därme zeigte sich nach der Enteiweißung immer eine starke Biuretreaktion. Als ich nun nach den bekannten Aminosäuren suchte, zeigte sich, daß Phosphorwolframsäure zunächst keine Fällung gab. Nach einigen Stunden setzte sich ein minimaler Niederschlag $a b$, der aber gar nicht das Aussehen der Basenniederschläge hatte. Von den Farbenreaktionen fiel die Millonsche negativ aus, was freilich wegen des Salzgehaltes der Flüssigkeit nicht viel beweist, aber auch die Paulysche Diazoreaktion war oft auffallend schwach. Es mußte also, abgesehen von der Spaltung des Peptons, noch weiteres vorgegangen sein.

Um zu prüfen, ob eine Desamidierung eingetreten sei, suchte ich nach etwa vorhandenen flüchtigen Basen. Ich versetzte einen Teil der Flüssigkeiten mit Baryumcarbonat ${ }^{1}$ ) und destillierte sie $a b$, indem ich das Destillat in $\mathrm{n} / 10$-Schwefelsäure auffing. In der Tat zeigte sich, daß bei allen Versuchen eine beträchtliche Menge einer flüchtigen Base, also vermutlich Ammoniak, vorhanden war. Ganz wie es Hart beschreibt, mußte auch hier die vorgelegte Schwefelsäure vor dem Titrieren aufgekocht werden. In einem Versuch z. B., in dem die Därme von 10 kleinen Fischen in die bluthaltige Ringersche Lösung kamen, wurde eine Peptonmenge, die $440 \mathrm{mg} \mathrm{N}$ enthielt, in die Därme

1) E. Hart, Diese Zeitschrift, Bd. XXXIII, S. 353 (1901). 
eingeführt. In der enteiweißten Außenflüssigkeit waren, an einer Probe bestimmt, bevor die Därme hereinkamen, $7 \mathrm{mg} \mathrm{N}$ und kein Ammoniak vorhanden, 7 Stunden später gab das enteiweißte Filtrat eine schwache Biuretreaktion. Es betrug $884 \mathrm{ccm}$.

$50 \mathrm{ccm}$ nach Kjeldahl verascht, sättigten $13,1 \mathrm{ccm} \mathrm{n/10-}$ $\mathrm{SO}_{4} \mathrm{H}_{2}$ ab, $884 \mathrm{ccm}$ enthielten also $336 \mathrm{mg} \mathrm{N}$.

$100 \mathrm{ccm}$, mit Baryumcarbonat destilliert, sättigten $\mathrm{ab}$ $4,4 \mathrm{ccm} n / 10-\mathrm{SO}_{4} \mathrm{H}_{2}$. Also enthielten, auf Ammoniak berechnet, die $884 \mathrm{ccm} 54 \mathrm{mg}$ Ammoniak-N.

Im Innern der Därme waren nach der Enteiweißung noch $147 \mathrm{mg} \mathrm{N}$ vorhanden. Der 4. Teil sättigte, mit Baryumcarbonat destilliert, $1,1 \mathrm{ccm}$ n/10-Säure $\mathrm{ab}$, also waren $6 \mathrm{mg}$ Ammoniak vorhanden.

Die anderen Versuche hatten folgende Ergebnisse:

1 Darm. Außen $10 \mathrm{mg} \mathrm{N}$, 2,5 mg Ammoniak-N. Keine Biuretreaktion:

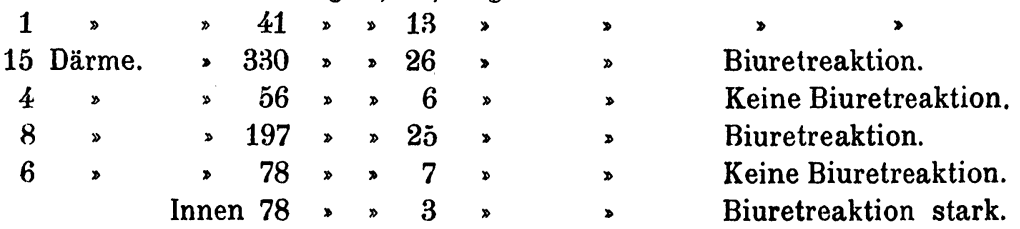

Dazu kommen 2 Versuche, bei denen die mit Muscheln vollgestopften Därme frischgefangener Fische in die bluthaltige Ringersche Lösung kamen.

4 Tiere. Vor Hereinlegen der Därme enthielt die Außenflüssigkeit, an einer Probe bestimmt, im enteiweißten Filtrat $1,1 \mathrm{mg} \mathrm{NH}_{3}-\mathrm{N}$. Nach 6 Stunden waren im Filtrat vorhanden $96 \mathrm{mg} \mathrm{N}$ und $8,5 \mathrm{mg} \mathrm{NH}_{3}-\mathrm{N}$.

5 Tiere. Die Därme sind halb gefüllt. Es wird noch etwas Pepton hereingefüllt. Die Außenflüssigkeit enthält anfangs $10 \mathrm{mg} \mathrm{N}$ im Filtrat und kein Ammoniak. Nach 9 Stunden wird sie unter Zusatz von Essigsäure koaguliert. Das Filtrat beträgt $375 \mathrm{ccm} .37 \mathrm{ccm}$, nach Kjeldahl verascht, sättigen ab $8,1 \mathrm{ccm} \mathrm{n} / 10-$ Säure, also $114 \mathrm{mg} \mathrm{N}$. $320 \mathrm{ccm}$, mit $\mathrm{BaCO}_{3}$ destilliert, sättigen ab 7,7 ccm n/10-Säure, also $10,3 \mathrm{mg} \mathrm{N}$. Im Innern der Därme finden sich $44 \mathrm{mg} \mathrm{N}, 2 \mathrm{mg}$ Ammoniak. Daß also aus den mit Pepton oder Muscheln gefüllten 
Därmen neben anderen stickstoff haltigen Substanzen auch eine flüchtige Base, vermutlich Ammoniak, übergegangen war, daran konnte kein Zweifel sein. Es fragte sich, ob das Ammoniak aus den Därmen oder aus dem Pepton stammte. Ich habe dreimal Därme, nur mit Ringerscher Lösung gefüllt, zu den Versuchen verwendet.

2 Därme, mit Ring erscher Lösung gefüllt. Außen Ring ersche Lösung mit besonders viel Blut. Bei Beginn des Versuches enthielten $45 \mathrm{ccm} \mathrm{4,6} \mathrm{mg} \mathrm{N,} \mathrm{nach} 7$ Stunden $50 \mathrm{ccm} \mathrm{4,8} \mathrm{mg} \mathrm{N}$.

1 Darm eines großen Fisches, mit Ringerscher Lösung gefüllt. In der Außenflüssigkeit ist nach 6 Stunden kein Ammoniak vorhanden.

1 Darm, mit Ring erscher Lösung gefüllt, nach 11 Stunden noch gut beweglich. Die gesamte Außenflüssigkeit sättigt bei der Destillation mit $\mathrm{BaCO}_{3} 0,6 \mathrm{ccm}$ n/10-Säure ab, also nur Spuren Ammoniak.

Das Ammoniak stammte also aus dem Pepton oder den Muscheln.

Ferner war die Frage, ob das Ammoniak durch einfache fermentative Spaltung aus den Eiweißkörpern entstanden, und ob es etwa durch Bakterien gebildet war. Ich habe daher dreimal eine Anzahl Därme frisch aus dem Tiere genommen, zerschnitten, mit Sand zerrieben, und den Brei mit Pepton zusammen zu der bluthaltigen Ringerschen Lösung hinzugesetzt und Sauerstoff hindurchgeleitet.

4 Därme. 6-7 Stunden. Die Flüssigkeit wird koaguliert, das Filtrat enthält $496 \mathrm{mg} \mathrm{N}$, aber nur $6 \mathrm{mg}$ Ammoniak-N.

2 Därme. Blut, Ringersche Lösung, Pepton und Darmextrakt werden gemischt. Die eine Hälfte wird sofort koaguliert; sie enthält $141 \mathrm{mg} \mathrm{N}$ und 2,5 $\mathrm{mg} \mathrm{NH}_{3}-\mathrm{N}$. Durch die andere Hälfte wird Sauerstoff geleitet; sie enthält dann nach der Enteiweißung $137 \mathrm{mg} \mathrm{N}$ und $3,4 \mathrm{mg} \mathrm{NH}_{3}-\mathrm{N}$.

4 Därme. Statt des Peptons wird Pepton hinzugesetzt, das mehrere Wochen mit Chloroform versetzt im Brutschrank mit Darmextrakt gestanden hatte. Das Chloroform wird entfernt, Ringersche Lösung, der Brei der 4 Därme und Blut hinzugefügt und $10 \frac{1 / 2}{2}$ Stunden Sauerstoff durchgeleitet. Im 
enteiweißten Filtrat waren $84 \mathrm{mg} \mathrm{N}$ und $5,4 \mathrm{mg}$ Ammoniak-N vorhanden.

Bei allen diesen Versuchen wurde wie ja bei jeder fermentativen Eiweißspaltung Ammoniak gefunden, aber entschieden weniger als bei den Versuchen am überlebenden Darm. Ich glaube diese Resultate nicht anders deuten zu können, als daß beim Durchtritt durch die Darmwand eine Desamidierung der Eiweißspaltungsprodukte eintritt. Daß die "überlebenden Organe " andere Eigenschaften haben, als die Extrakte der Organe, ist ja auch sonst, besonders von der Leber, wohl bekannt.

Die nächste Aufgabe war nun natürlich, einzelne Aminosäuren durch die Därme resorbieren zu lassen. Da aber die Zeit meines Neapeler Aufenthaltes zu Ende ging, so habe ich nur noch 3 Versuche mit Lysin und Asparaginsäure ausführen können. Das Lysin hat mir teils Herr Dr. Henze liebenswürdig zur Verfügung gestellt, es stammte von einer Säurespaltung von Koralleneiweiß, teils habe ich es aus Gelatine nach der Methode von Kossel und Kutscher dargestellt. Die Asparagin säure war ein Kahlbaumsches Präparat.

3 Därme von großen Fischen. Innen $14 \mathrm{ccm}$ dünne $\mathrm{NaHCO}_{3}$-Lösung, in der sich 0,25 g Asparaginsäure gelöst hatten. Dauer 6 Stunden. Im enteiweißten Filtrat der Außenflüssigkeit. fanden sich $27 \mathrm{mg} \mathrm{N}$, davon $9 \mathrm{mg}$ in Form von Ammoniak.

4 Därme. Eingeführt 0,23 g Lysincarbonat. Nach dem Enteiweißen sind $215 \mathrm{ccm}$ Außenflüssigkeit vorhanden.

$10 \mathrm{ccm}$ sättigen nach $\mathrm{Kjeldahl}$ verascht $1,5 \mathrm{ccm}{ }^{\mathrm{n}} /{ }_{10^{-}}$ Säure $a b$. Das sind $45 \mathrm{mg} \mathrm{N}$.

$200 \mathrm{ccm}$ sättigen mit Baryumcarbonat destilliert $10 \mathrm{ccm}$ n/ ${ }_{10}$-Säure ab. Das sind $15 \mathrm{mg}$ Ammoniak-N.

5 Därme von kleinen Tieren. Mit Lysinchlorid gefüllt. 10 Stunden. Filtrat der Außenflüssigkeit $240 \mathrm{ccm} .25 \mathrm{ccm}$ sättigen, nach $\mathrm{Kjeldahl}$ verascht, $2,6 \mathrm{ccm} \mathrm{n} /{ }_{10}$-Säure $a b$. Das sind $35 \mathrm{mg} \mathrm{N}$.

$215 \mathrm{ccm}$ sättigen, mit $\mathrm{BaCO}_{3}$ verascht, $3,1 \mathrm{ccm}$ n/ ${ }_{10}$-Säure ab. Das sind 4,7 mg Ammoniak-N.

Eine Desamidierung tritt also auch hier ein. Eine genauere 
Untersuchung war bei den kleinen Mengen nicht möglich. Ich hoffe aber, die Versuche bald wieder aufnehmen zu können.

. So wenig die Versuche abgeschlossen sind, ergibt sich aus ihnen, das bei den Fischen bereits beim Passieren der Darmwand die Eiweißspaltungsprodukte teilweise desamidiert werden, in Ammoniak und einen, zunächst noch unbekannten Rest zerfallen. Diese Tatsache fügt sich gut zu mehreren schon bekannten. Einmal haben Nencki und seine Mitarbeiter ${ }^{1}$ ) in dem von dem tätigen Darm abströmenden Blut viel Ammoniak gefunden, und zweitens hat Neubauer ${ }^{2}$ ) im ganzen Organismus eine Desamidierung der Aminosäuren als den Anfang ihrer Umwandlung gesehen.

1) M. Nencki, J. P. Pawlow und J. Zaleski, Arch. f. experim. Pathol. u. Pharmakol., Bd. XXXVII, S. 26 (1898). - M. Nencki und J. P. Pawlow, ibid., Bd. XXXVIII, S. 215 (1898). - S. S. Salaskin, Diese Zeitschrift, Bd. XXV, S. 448 (1898). - Horodynski, Salaskin und Zaleski, ibid., Bd. XXXV, S. 246 (1902).

2) 0. Neubauer, Deutsch. Arch. f. klin. Med., Bd. XCV, S. 211 (1909). 